The relative risks of fetal and infant death associated with pregestational diabetes were 4.54 (95\%CI: 3.41-6.05, p < 0.0001) and 1.82 (95\%CI: $0.98-3.38, p=0.06)$ respectively. The odds of a fetal or infant death increased by $19 \%(\mathrm{OR}=1.19,95 \% \mathrm{CI}: 1.02-1.39$, $\mathrm{p}=0.02)$ and $42 \%(\mathrm{OR}=1.42,95 \% \mathrm{CI}: 1.09-1.85, \mathrm{p}=0.01)$ respectively for each percentage increase in peri-conception $\mathrm{HbA} 1 \mathrm{c}$, although third trimester $\mathrm{HbA1c}$ was a stronger predictor of late fetal death $(\mathrm{OR}=1.67,95 \% \mathrm{CI}: 1.25-2.24, \mathrm{p}=0.001)$.

Conclusions Pre-gestational diabetes is associated with a substantially increased risk of fetal death in normally-formed offspring. The effect is largely moderated by glycaemic control, with increasing $\mathrm{HbA1c}$ conferring higher risks of both fetal and infant death.

\section{PP.18 CAESAREAN SECTION AND TIME TO NEXT BIRTH, ECTOPIC PREGNANCY, MISCARRIAGE OR STILLBIRTH-A DANISH REGISTER-BASED STUDY}

doi:10.1136/archdischild-2013-303966.299

${ }^{1} \mathrm{SM}$ O'Neill, ${ }^{3} \mathrm{E}$ Agerbo, ${ }^{2} \mathrm{LC}$ Kenny, ${ }^{\mathrm{T}} \mathrm{TB}$ Henriksen, ${ }^{5} \mathrm{PM}$ Kearney, 'RA Greene, ${ }^{3} \mathrm{~PB}$ Mortensen, ${ }^{1} \mathrm{JE}$ Lutomski, AS Khashan, ${ }^{2}$ S Meaney. ' National Perintal Epidemiology Centre, University College Cork, Cork, Ireland; ${ }^{2}$ Anu Research Centre, University College Cork, Cork, Ireland; ' National Centre for Register-based Research, Arrhus University, Aarhus, Denmark; ${ }^{4}$ Perinatal Epidemiological Research Unit, Arrhus University Hospital, Aarhus, Denmark; ${ }^{5}$ Department of Epidemiology and Public Health, University College Cork, Cork, Ireland

Aim Estimate time to next birth, risk of miscarriage, ectopic pregnancy or stillbirth in women with primary Caesareans.

Methods Danish population-registry cohort of births from 19822010 ( $n=833,162)$. Analysis using Cox-regression models.

Results Prior Caesarean group had longer birth intervals. No increased risk of ectopic pregnancy or miscarriage, but significantly increased risk of stillbirth among women with a prior Caesarean.

Conclusion Prior Caesareans were associated with an increased birth interval and increased risk of stillbirth compared to vaginal deliveries.

\section{Abstract PP.18 Table}

\begin{tabular}{lllll}
\hline & Birth Interval & Ectopic & Miscarriage & Stillbirth \\
\hline Delivery & adj.HR $(95 \% \mathrm{Cl})$ & & & \\
Vaginal & 1 & & & \\
Instrumental & $1.08(1.07,1.09)$ & $0.91(0.86,0.96)$ & $1.03(1.01,1.05)$ & $0.95(0.84,1.08)$ \\
Elective CS & $0.83(0.82,0.84)$ & $1.02(0.95,1.09)$ & $0.92(0.90,0.95)$ & $1.10(0.94,1.30)$ \\
Acute CS & $0.89(0.88,0.90)$ & $1.03(0.99,1.0)$ & $0.98(0.96,1.00)$ & $1.16(1.04,1.28)$ \\
MRCS & $0.64(0.60,0.69)$ & $1.04(0.69,1.57)$ & $0.78(0.66, .092)$ & $0.54(0.13,2.18)$ \\
\hline
\end{tabular}

Instrumental: vacuum/forceps; CS:Caesarean-section; MRCS: Maternally-Requested CS

\section{PP.19 WITHDRAWN BY AUTHOR}

\section{PP.20 THE POTENTIAL OF GENERAL PRACTISE DATA FOR CONGENITAL ANOMALY RESEARCH}

doi:10.1136/archdischild-2013-303966.300

R Sokal, K Fleming, LT Tata. University of Nottingham, Nottingham, UK

Background General practise data provide large population-based cohorts of individuals with prospectively collected medical information with promising potential for studying the causes and consequences of congenital anomalies. We sought to validate these data through comparison with congenital anomaly registries.

Methods Our study population was 794,209 children in The Health Improvement Network (THIN) primary care database, born between 1990 and 2009 with a median follow-up of 6.7 years. We compared the birth prevalence of any major and system-specific congenital anomalies with the European Surveillance of Congenital Anomalies (EUROCAT) United Kingdom registries.

Results The birth prevalence of any major congenital anomaly for children in THIN diagnosed before one year of age was 198 per $10,000(95 \%$ CI 195 - 201) which was slightly higher than the EUROCAT prevalence of 167 per 10,000 (Relative Risk 1.18, 95\% Confidence Interval $1.16-1.20)$. Absolute differences in prevalence between THIN and EUROCAT were small across 16 system-specific anomaly groups. The majority of children in THIN with major congenital anomalies had recorded diagnoses before 1 year of age (72\%), but including children diagnosed at any age increased the overall prevalence to 277 per 10,000 births.

Conclusions The prevalence of congenital anomalies in THIN was consistent with EUROCAT for early diagnoses, demonstrating THIN to be a valid source for investigating congenital anomalies. Age of diagnosis is an important factor in explaining a higher overall prevalence in THIN; the inclusion of diagnoses made after one year of age substantially improves capture of diagnoses making THIN more complete than registry data.

\section{PP.21 THE EFFECT OF FACTORS OF SOCIAL EXCLUSION ON ACCESS TO ANTENATAL CARE AND THE SUBSEQUENT IMPACT ON FETAL OUTCOMES}

doi:10.1136/archdischild-2013-303966.301

EM Mercer, DOC Anumba. University of Sheffield, Sheffield, UK

Background Delayed access to antenatal care is linked to maternal deaths and morbidity. Social deprivation also accounts for much maternal and child ill-health, with national health data showing discrepancies in the access and utilisation of care by groups of women who differ by ethnicity, age and socioeconomic status.

Objectives To map the prevalence of social deprivation and delayed access to antenatal care amongst women attending a regional referral maternity service and to correlate with fetal outcomes.

Methods Retrospectively analysed data collected from 59,847 singleton births at the Jessop Wing Hospital, Sheffield, UK, between 2002 and 2010, identifying maternal demographic features and neonatal outcomes. We plotted the Index of Multiple Deprivation (IMD) scores of women against the distribution of delayed access to antenatal care.

Results The geographic distribution of high deprivation scores and first pregnancy care attendance after 20 weeks gestation matched closely. High IMD scores increased the risk of being a late booker (OR: 1.092, 95\% CI: 1.01-1.18, $\mathrm{p}=0.031$ ) and being of minority ethnic extraction (OR: 5.6, 95\% CI: 5.2-5.9, p < 0.001), and significantly predicted low birth weight (OR: $1.66,95 \% \mathrm{CI}$ : 1.31-2.12, $\mathrm{p}<0.001$ ), premature delivery (OR:1.34, 95\% CI: 1.06 $1.70, p=0.017)$ and stillbirth (OR: $2.25,95 \% \mathrm{CI}: 1.68-3.01$, $\mathrm{p}<0.001)$. When sociodemographic variables were adjusted for, late booking did not independently predict adverse maternal or fetal outcomes.

Conclusions Disparities remain for women from certain backgrounds. Geographic mapping of high deprivation scores can direct research and health delivery strategies seeking to promote better access to pregnancy care and mitigate the resulting adverse fetal outcomes.

\section{PP.22 ANTEPARTUM HAEMORRHAGE OF UNKNOWN ORIGIN: SHOULD WE BE WORRIED?}

doi:10.1136/archdischild-2013-303966.302

S Bhattacharya, S Bhandari, EA Raja. University of Aberdeen, Aberdeen, UK 